\title{
Politique
}

\section{Intelligentsia et médias : De l'éducation populaire au Pouvoir}

\section{Frank Desoer}

Volume 1, numéro 2, automne 1982

Les médias et les pouvoirs

URI : https://id.erudit.org/iderudit/040403ar

DOI : https://doi.org/10.7202/040403ar

Aller au sommaire du numéro

Éditeur(s)

Société québécoise de science politique

ISSN

0711-608X (numérique)

Découvrir la revue

Citer cet article

Desoer, F. (1982). Intelligentsia et médias : De l'éducation populaire au Pouvoir. Politique, 1(2), 97-116. https://doi.org/10.7202/040403ar d'utilisation que vous pouvez consulter en ligne.

https://apropos.erudit.org/fr/usagers/politique-dutilisation/ 


\title{
Intelligentsia et medias : De l'éducation populaire au Pouvoir
}

\author{
Frank Desoer \\ Université de Montréal
}

La Révolution tranquille correspond à une phase historique d'adaptation durant laquelle la société québécoise s'est dotée d'instruments politiques conformes à son stade de développement économique et social. Mais ce changement avant de se traduire en gestes politiques concrets a suivi un long processus de gestation. Avant leur application durant les années soixante, les réformes ont dû être jugées souhaitables par une bonne fraction de la population. Il a donc fallu qu'elles soient préalablement pensées, formulées et que leurs objectifs soient largement diffusés auprès du public.

Aussi, l'après-guerre marque une étape importante dans la modernisation de la société québécoise. Non seulement le Québec s'industrialise à un rythme rapide, mais il connaît de plus une profonde mutation sur le plan des idées. Les multiples revendications et initiatives venant du monde syndical, coopératif, universitaire et artistique préfigurent déjà les grandes orientations politiques que notre société va se donner plus tard. On peut penser, du reste, que c'est à cette époque de bouillonnement culturel, que le changement, dans son sens véritable, se produit au Québec. La Révolution tranquille ne représenterait, en fait, que le couronnement des efforts déployés pendant des 
années par les groupes les plus dynamiques de la société. Dans une large mesure, les jeux étaient faits avant la victoire électorale de l' «Équipe du Tonnerre » en 1960.

Durant l'après-guerre, certains acteurs s'illustrent particulièrement comme catalyseurs de changements. Outre le mouvement syndical qui constitue certes le centre de contestation le plus cohérent du duplessisme, ceux que l'on pourrait appeler les «nouveaux intellectuels» jouent un rôle de premier plan. Les années quarante et cinquante verront, en effet, l'ascension d'une nouvelle génération d'élites - sociologues, économistes, spécialistes de l'éducation... - qui se distingue par un savoir spécialisé et «concret» acquis à l'Université. S'inspirant d'analyses économiques et sociales du milieu québécois, cette intelligentsia généralement moderniste et fédéralisante, entreprend la critique systématique de l'idéologie traditionnelle et de la culture québécoise. Elle se manifeste dans certaines institutions, revues et mouvements, particulièrement l'École des Sciences Sociales de l'Université Laval, la revue Cité Libre et dans certains cercles d'études comme l'Institut Canadien des Affaires Publiques ${ }^{1}$.

Les «nouveaux intellectuels» durant l'après-guerre se donnent comme tâche de faire l'éducation civique et démocratique d'un peuple jugé profondément immoral. Par là, ils cherchent à créer un consensus social autour des grands objectifs politiques et sociaux de la Révolution Tranquille.

En prêchant la modernisation de la société québécoise, la nouvelle intelligentsia travaille en même temps à sa propre ascension en tant que catégorie sociale. Elle défend un modèle, de société qui est appelé à conférer aux détenteurs de l'expertise économique et sociale, un pouvoir et une légitimité dont ils étaient privés dans la société sclérosée des années Duplessis.

1. Voir: Marcel Rioux, "Sur l'évolution des idéologies au Québec», Revue de l'Institut de Sociologie, 1968, Bruxelles, 95-124. 
Avec la Révolution Tranquille, certains intellectuels, abandonnant leur rôle d'intellectuels critiques, deviendront d'ailleurs les premiers technocrates de l'État québécois. La fonction publique québécoise connaît, on le sait, une évolution quantitative et qualitative très importante durant les années soixante. Entre 1964 et 1971 , le nombre d'universitaires à l'emploi des ministères se multiplie par $120 \%$. «Ce mouvement s'enrichit d'un bond spectaculaire des occupations à teinte techno-bureaucratique». À l'intérieur de celles-ci, «l'ingénierie humaine et socio-économique (agents de la planification étatique, spécialistes des sciences de l'éducation...), inexistante avant 1955, s'accroît de $420.5 \%{ }^{2}$.

Aussi, suivant Gramsci, on pourrait dire que l'on assiste au tournant des années cinquante et soixante au transfert du pouvoir des «intellectuels traditionnels» aux «intellectuels organiques».

Divers moyens s'offrent aux nouvelles élites pour mener à bien leur mission de «réforme morale et intellectuelle». Parmi ceux-là, les mass-média et particulièrement la télévision, apparue en 1952, s'imposent par leur efficacité d'autant plus que Radio Canada a le monopole jusqu'au début des années soixante.

\section{L'apolitisme d'une génération}

Lorsque l'on dresse le bilan des années du duplessisme, on peut s'étonner de ce que les intellectuels progressistes soient restés alors «en marge du politique». Comment expliquer, en effet, qu'ils n'aient pas lutté plus «concrètement» contre un régime qu'ils accusaient par ailleurs de tous les maux du monde?

2. Jean-Jacques Simard, La longue marche des technocrates, Éditions Coopératives Albert St-Martin, 1979, 36-37. 
Gérard Bergeron a été, à cet égard, l'un des critiques les plus vigoureux des intellectuels de sa génération, celle qu'il qualifie de génération de «l'impuissance politique». Il condamnera à la fin des années cinquante, ceux qui, préferant investir leurs énergies dans des mouvements socio-éducatifs à la périphérie de la «politique qui se fait», rechignent à s'engager dans une action visant directement la gouverne ${ }^{3}$.

De fait, pour les intellectuels de l'époque, les partis politiques traditionnels n'offrent rien de très excitant. Le parti libéral n'apparaît pas tellement différent de l'Union Nationale. Ils pratiquent tous deux le même style «politicailleur». On s'engage plutôt dans le mouvement syndical (surtout CTCC), les ligues de moralité publique, les mouvements d'éducation populaire (ICEA, par exemple) et le journalisme (Cité Libre et «le Devoir»)...

La seule expérience de parti politique impliquant réellement les intellectuels à l'époque est celle du «Rassemblement » et elle s'avère être un échec complet.

Parmi ses fondateurs et ses dirigeants, on retrouve la fine fleur de la nouvelle élite: Pierre Dansereau, doyen de la Faculté des Sciences Sociales de l'Université de Montréal, PierreElliott Trudeau, avocat, Jean-Paul Lefebvre, directeur-adjoint de la CTCC, Jacques Hébert, directeur de l'hebdomadaire «Vrai», Gérard Pelletier, commentateur de radio et de télévision, Arthur Tremblay, professeur de pédagogie à l'Université Laval ${ }^{4}$.

Le Rassemblement, en proie à des dissensions idéologiques internes, n'a jamais pu définir une ligne d'action précise. En fait, malgré beaucoup de bonne volonté, le Rassemblement ne rassemblait personne... Pris, 1971.

3. Gérard Bergeron, $D u$ duplessisme à Trudeau et Bourassa, Montréal, Parti

4. Roch Denis, Lutte de classes et question nationale au Québec (1948-1968), Montréal, Presses Socialistes Internationales, 1979, 189. 
Il semblerait, en fait, que la nouvelle élite n'ait jamais eu de projet politique substantiel à proposer. Dans un article désormais célèbre ${ }^{5}$, Marcel Rioux exprime clairement la chose: l'idéologie de ces intellectuels se définit avant tout par la «négative». Cité Libre, par exemple, s'attache beaucoup plus à dénoncer les mythes de l'idéologie traditionnelle et la collusion des élites politiques et cléricales qu'à formuler des alternatives nouvelles.

En réalité, cette attitude qui peut sembler tenir de l'incohérence, du manque de prise sur le réel voire même de la faiblesse des nouvelles élites, prend un sens différent lorsqu'on la resitue dans la démarche intellectuelle qui était la sienne.

Pour la plupart des intellectuels progressistes, l'obstacle principal à la démocratie au Québec réside dans «l'inconscience populaire». Pierre Elliott Trudeau écrivait dans Cité Libre:

"L'immoralisme électoral et civique des Canadiens français, leur penchant pour l'autoritarisme, les thèses anti-démocratiques qu'ils apprennent au collège, les structures non-adultes où ils se débattent à l'université, le peu de place qu'ils occupent comme laïcs dans l'Église québécoise, les cadtes sociaux étroits où ils vivent dans les campagnes, les positions subalternes qu'ils occupent dans les structures autoritaires du capitalisme, leur crainte de recourir à l'État qui, pourtant seul, pourrait donner à la collectivité les moyens de sortir de son marasme, le peu de cas qu'ils font (dans l'ensemble) des atteintes à la liberté de parole, de presse et d'association, tout cela constitue autant de caractéristiques d'un peuple qui n'a pas encore appris à se gouverner lui-même, d'un peuple où la démocratie ne peut pas être prise pour acquise ${ }^{6}$.

En conséquence, la lutte pour une société démocratique s'impose comme tâche prioritaire. À un problème moral correspond un remède moral:

5. Marcel Rioux, op. cit.

6. Voir Pierre-Elliott Trudeau, «Un manifeste démocratique», Cité Libre, no. 22, octobre $1958,17-21$. 
«Il nous faut, disait Bergeron, une vaste campagne d'éducation démocratique dont l'objectif sera de «sensibiliser une génération politique aux valeurs essentielles d'un civisme actif et toujours attentif (...). Il faut faire du Canadien français qui ne s'est guère considéré que comme un sujet prébendé ou un national en danger, un citoyen actif qui a un rôle à jouer dans la recherche de son mieux-être social » ${ }^{7}$.

Dénonçant le «mensonge érigé en système, la vénalité, le goût croissant pour une servitude bien nourrie», les abbés Dion et O'Neill «concluent également à l'urgence de procéder à un travail d'éducation morale et civique de la population ${ }^{8}$.

La logique qui sous-tend ce raisonnement prend pour acquis que la réforme politique et sociale passe d'abord et avant tout par la réforme des consciences individuelles. Une saine démocratie repose sur la «conscience formée et informée du citoyen». Celui-ci, pour avoir vraiment prise sur le réel, doit d'abord avoir "conscience de lui-même, de son environnement et des finalités de son action ${ }^{9}$. Le citoyen jouissant des lumières de la «Raison» est le meilleur garant du respect des institutions démocratiques. "Il ne peut y avoir de "grande politique» dans quelque domaine que ce soit, disait Jean-Louis Gagnon, si le peuple n'y croit et s'il n'accepte pas de s'y associer. Du moins en démocratie puisque tout gouvernement élu doit s'assurer toujours du «consensus» ou de l'accord d'une vaste majorité ${ }^{10}$.

Ainsi, la réforme des structures politiques et sociales passe par une réforme préalable de la culture. Ce raisonnement se situe à l'inverse de celui que vont développer plus tard les ani-

7. Gérard Bergeron, op. cit., 116.

8. Gérard Dion, Louis O'Neill, "L'immoralité politique dans la province de Québec », in Gérard Boismenu, Laurent Mailhot, Jacques Rouillard, Le Québec en textes, Montréal, Boréal Express, 1980, 160-167.

9. André-J. Bélanger, Ruptures et Constantes, Montréal, Hurtubise $\mathrm{HMH}$, 1977,75 .

10. Jean-Louis Gagnon, «Préface», in Institut Canadien des Affaires Publiques. L'utilisation des ressources bumaines, Montréal, Éditions du Jour, 1965, 16. 
mateurs de «Parti Pris». Dans la perspective de ces derniers, ce sont les structures économiques et politiques de la colonisation qui sont à la base de l'aliénation collective.

L'action des intellectuels d'après-guerre prend un relief particulier étant donné ce qui précède. On peut, en effet, émettre l'hypothèse que l'engagement social et éducatif apparemment en marge du politique soit, en fait, éminemment politique dans ses intentions et ses finalités.

Un bref coup d'œil sur les activités des nouvelles élites contribue à nous le démontrer.

Le «réseau » des intellectuels d'après-guerre

L'une des caractéristiques intéressantes des «nouveaux intellectuels» est la remarquable homogénéité de leur formation et de leurs activités. Dans le contexte difficile du duplessisme, les institutions, revues et mouvements où ils se regroupent sont peu nombreux. Il est donc relativement facile de reconstituer ce que l'on pourrait appeler le «réseau» de l'intelligentsia montante.

\section{Formation d'un esprit critique}

Nous l'avons signalé plus tôt, la Faculté des Sciences Sociales de Laval et la revue Cité Libre constituent les pôles de rassemblement et de contestation les plus importants pour toute une nouvelle génération d'intellectuels.

Mais s'il fallait établir un point de départ au «réseau», les mouvements d'action catholique s'imposeraient d'emblée. Ils constituent réellement, durant les années quarante, le berceau d'une large fraction des nouveaux intellectuels. Certains d'entre eux en ont même été les principaux dirigeants durant leur jeunesse. C'est le cas par exemple des Jeanne Sauvé, Maurice Pinard, Guy Cormier et Marc Lalonde. 
André Raynauld a été, en tant que fondateur en 1950 de la Fédération des Coopératives Étudiantes (JEC) et secrétaire-général de l'U. de $M$., un leader étudiant très actif.

$\mathrm{La}$ formation intellectuelle reçue dans les mouvements d'action catholique semble avoir fortement pénétré l'esprit des nouvelles élites. Ainsi, elles restent longtemps marquées par la pensée catholique sociale d'inspiration européenne. Tout en conservant un certain attachement à la présence de l'Église dans la société, elles seront très critiques vis-à-vis le cléricalisme. De fait, le volontarisme du projet jéciste «d'action rationnelle sur un milieu concret» constitue déjà une rupture avec l'idéologie dite «dominante» ${ }^{\mathbf{1 1}}$. Il fournit aux «nouveaux intellectuels» un premier cadre de référence idéologique à la contestation des pouvoirs en place.

La naissance des facultés de sciences sociales à Montréal et à Québec a certes contribué, elle aussi, à la formation d'esprits critiques. En renouvelant la perspective de la recherche qui doit s'attaquer désormais aux «faits sociaux pris comme tels" dans l'étude des interactions entre l'individu et la société, l'Université s'oppose au savoir abstrait et normatif véhiculé jusque là par la doctrine officielle et l'École Sociale Populaire. Elle tente, par là, de s'ajuster aux besoins sociaux en termes de recherche et de préparation d'un personnel qualifié dans les domaines des relations industrielles, du service social, de la recherche sociologique et économique. Ces domaines accusaient, durant l'après-guerre, un retard dont le coût social s'avérait considérable.

L'École des Sciences Sociales de l'Université Laval sous la direction éclairée du Père Georges-Henri Lévesque a été le foyer de plusieurs pionniers de la connaissance sociale au Québec. Les travaux des Jean-Charles Falardeau, Maurice Tremblay, Fernand 
Dumont, Albert Faucher et Léon Dion ont nourri une prise de conscience des problèmes économiques et sociaux que posait l'industrialisation au Québec. Ils permettaient de prendre toute la mesure des responsabilités du pouvoir politique et clérical dans «la dégradation du capital humain» ${ }^{12}$.

On comprend, dès lors, que l'Université se soit attiré les foudres de Duplessis. Comme l'indique Jean-Charles Falardeau:

«Toute faculté de sciences sociales en tant que laboratoire d'observations et lieu de réflexion est source d'exaspération pour les idéologie traditionnelles dont chacune veut l'inféoder... ${ }^{13}$.

\section{L'essor de l'éducation populaire}

Falardeau écrivait encore que «la Faculté possède un devoir de présence à tous ceux qui peuvent avoir besoin d'elle ${ }^{14}$.

L'engagement social de la Faculté, élément très nouveau à l'époque au Québec, est certes impressionnant. Elle collabore à de très nombreux organismes et associations dont le mouvement ouvrier qui a alors grand besoin d'économistes et de conseillers.

En 1951, l'Université Laval crée un service extérieur d'éducation sociale, «Le Centre de Culture Populaire», qui anime des stages d'études et effectue des enquêtes sociales. Il existe, à l'École des Sciences Sociales de Laval ainsi qu'à l'École des Hautes Études Commerciales, une longue tradition de cours du soir qui, dans les années cinquante, s'étend de manière très importante ${ }^{15}$.

12. Voir: notamment Jean-Charles Falardeau, L'essor des sciences sociales au Canada français, Québec, Ministère des Affaires culturelles, 1964 et Essais sur le Québec contemporain, Québec, PUL, 1953.

13. Jean-Charles Falardeau, "Lettre à mes étudiants à l'occasion des vingt ans de la Faculté des Sciences Sociales de Québec », Cité Libre, no. 23, mai 1959.

14. Ibid.

15. Jean-Louis Roy, La marche des Québécois. Le temps des ruptures (1945-1960), Montréal, Leméac, 1976, 293. 
L'éducation populaire dite permanente prend, de manière générale, un essor marqué durant l'après-guerre.

Dans le milieu syndical, cela se traduit par la mise sur pied en 1948 du service d'éducation de la CTCC sous la pression de Gérard Picard et de Jean Marchand. Ce service vise à donner une formation fonctionnelle aux membres et aux cadres du syndicat. Contrairement à l'enseignement abstrait prodigué jusque là par les religieux, on diffuse aux syndiqués des connaissances pratiques (techniques de négociation collective, code du travail...) ${ }^{16}$.

Parmi les animateurs principaux de l'éducation à la CTCC, on retrouve des piliers du réseau des intellectuels des années cinquante comme Gérard Pelletier (directeur du journal «Le Travail») et Jean-Paul Lefebvre (directeur-adjoint du service d'éducation). Ce dernier, dans un article publié dans la revue Cité Libre en 1955, met en évidence l'importance de «conscientiser» la «masse ouvrière»:

«Lorsque l'éducation sera vraiment dépouillée d'une certaine tradition individualiste et bourgeoise, le travailleur pourra faire la preuve que la culture, elle, y gagnerait à démocratiser ses méthodes et ses institutions $\gg{ }^{17}$.

Il devait ajouter plus tard que «la capacité productive des pauvres se situant en bonne part dans l'éducation» (...), la compréhension de l'homme de la rue est importante pour éviter que le progrès économique et social ne soit freiné par l'apathie et l'inertie des masses ${ }^{18}$.

Le service d'éducation de la CTCC met sur pied, en 1952, le «Collège Ouvrier» qui donne un enseignement en économie

16. Ibid., 87-95.

17. Jean-Paul Lefebvre, "L'éducation populaire au Canada français», Cité Libre, no. 13, novembre 1955, 21-23.

18. Jean-Paul Lefebvre, «Désormais seuls les sots prétendront avoir complété leur éducation», Institut Canadien des Affaires Publiques, op. cit., 133-134. 
(structure financière, fiscale, inflation, chômage...) et en politique (idéologies et structures sociales, changements technologiques...). De nombreux spécialistes des sciences sociales viennent y prodiguer leur savoir ${ }^{19}$.

\section{Le rôle stratégique des médias}

Inutile d'épiloguer sur la place de la revue Cité Libre dans la contestation du duplessisme et de l'idéologie «dominante».

Cette revue réunit à une certaine époque un aréopage impressionnant d'intellectuels progressistes. Outre Pierre-Elliott Trudeau et Gérard Pelletier, elle compte, parmi ses principaux animateurs, des universitaires de Laval (J.-C. Falardeau, Fernand Dumont, Léon Dion...), de Montréal (Albert Breton, André Raynauld, Pierre Dansereau...). On y retrouve, entre autres, beaucoup d'anciens de l'Action Catholique: Maurice Pinard, Marc Lalonde, Benoît Baril (premier président national de la JEC), Guy Cormier, Pierre Juneau, Jean-Paul Lefebvre...

Des journaux comme Vrai dirigé par Jacques Hébert, Le Devoir avec des journalistes comme Pierre Laporte et Gérard Filion, sont également sur la ligne de feu de la lutte antiduplessiste. Le Devoir s'est notamment illustré dans la dénonciation des pratiques de corruption du gouvernement. (voir, par exemple, «le scandale du gaz naturel» dévoilé en 1958). Tout comme Vrai, il attire d'ailleurs, dans son giron, plusieurs éléments de la nouvelle intelligentsia.

Mais on doit constater que si ces médias écrits de l'opposition possèdent un rayonnement certain parmi la couche intellectuelle, ils rejoignent assez difficilement le grand public. Aussi, les intellectuels se tournent-ils vers des médias qui apparaissent pouvoir diffuser plus largement leur message.

La radio éducative, par exemple, sert, durant l'aprèsguerre, de tremplin pour de nombreux éléments de la nouvelle

19. Jean-Louis Roy, op. cit., 94. 
élite. Elle prend, du reste, une expansion intéressante à l'époque. De 1941 à 1956, Radio-Collège produit plus de 3,860 émissions en coordination avec la Faculté des Arts de Laval et de Montréal. Ces émissions traitent des sujets les plus divers: théâtre, médecine, musique, science politique, psychiatrie... «Elles font éclater les garde-fous de la mobilité intellectuelle et idéologique autorisée au sein du système scolaire ${ }^{20}$.

De fait, avec le progrès économique et technologique et l'urbanisation accélérée que connait la société québécoise, aux lendemains de la guerre, il devient de plus en plus difficile pour les élites traditionnelles de maintenir leur emprise sur les réseaux de diffusion culturelle.

L'apparition de la télévision au Québec en 1952 joue un rôle important comme agent du changement culturel à la veille de la Révolution tranquille. Elle jouit, par rapport à la radio éducative, de la supériorité de la démonstration visuelle. Elle attire d'ailleurs vers elle beaucoup d'anciens collaborateurs de Radio-Collège. De plus, la société Radio-Canada, en tant qu'institution relevant du gouvernement fédéral, représentait une «oasis» pour la liberté d'expression dans le contexte étriqué du duplessisme.

Il existe, au début de la télévision, une mentalité de «participation communautaire» qui préside à l'élaboration de plusieurs émissions. Au Service des Émissions Éducatives de Radio-Canada, on se montre très soucieux d'associer diverses organisations sociales à la préparation d'émissions d'affaires publiques.

L'émission de télévision «les idées en marche» fournit un bon exemple à cet égard: animée par Gérard Pelletier, réalisée à la fin des années cinquante par Jean Pellerin, cette émission 
est organisée conjointement par le Service des Causeries de Radio-Canada et la Société Canadienne d'Éducation des Adultes. Des personnalités de divers milieux - SSJB, Action Catholique, HEC, Association Professionnelle des industriels - participent à sa préparation.

L'esprit qui animait cette série d'émissions est celui de l'éducation civique et populaire alors en vogue. Gérard Filion, dans un feuillet des «Idées en marche» souligne d'ailleurs que «ce qui importe d'abord, c'est de redonner au peuple canadienfrançais une juste notion de la politique et de la démocratie» ${ }^{21}$.

Durant les années cinquante, l'accessibilité de Radio-Canada aux nouvelles élites et aux organisations populaires dans lesquelles elles œuvrent, est particulièrement remarquable. Alors que Gérard Pelletier et Jean Marchand sont parmi les premières vedettes de la télévision (l'ex-secrétaire général de la CTCC aurait même été vu à trois émissions différentes durant une même journée!), des leaders de la Société Canadienne d'Éducation des Adultes comme Romain Desbois et Jean Charbonneau deviennent respectivement adjoint au service des recherches et réalisateur T.V. à Radio-Canada. D'anciens militants de l'Action Catholique comme Roméo Leblanc, André Patry, Maurice et Jeanne Sauvé font également un stage à Radio-Canada, soit en tant qu'animateurs ou réalisateurs.

Le cas de "l'Institut Canadien des Affaires Publiques».

Parmi tous les forums qui permettent un débat libre et ouvert durant l'après-guerre, l' «Institut Canadien des Affaires Publiques» (ICAP) occupe certes une place de choix.

Les conférences annuelles de l'ICAP (1954-1967) sont organisées avec le concours de l'Institut Démocratique Canadien et

21. Gérard Filion, in Les Idées en marche, Société Canadienne d'Éducation des Adultes, tiré de Roch Denis, op. cit., 187. 
de la Société Radio-Canada. Cette dernière voit non seulement au financement mais aussi au contenu des programmes de discussion. La diffusion des conférences à la radio commence dès 1954, puis à la télévision à partir de 1960. Ces discussions semblaient retenir l'intérêt d'un assez grand public puisqu'en moyenne, quelque 700 personnes allaient assister en personne aux conférences qui se tenaient chaque automne dans les Laurentides.

L'ICAP se veut un lieu de rencontre pour des hommes publics venant de secteurs très variés: entreprise privée, syndicalisme, enseignement, journalisme, fonction publique... Ses conférences fournissent simplement l'occasion d'un échange sur des problèmes généraux d'ordre politique, social et économique. Elles ne visent aucunement à ce que soient arrêtées des positions communes sur les questions débattues.

Les animateurs principaux de l'Institut sont: Léon Lortie (directeur de l'extension de l'enseignement à l'Université de Montréal), Napoléon Leblanc (directeur du Centre de Culture Populaire), Marcel Rioux, André Raynauld, Maurice et Jeanne Sauvé, Pierre Dansereau, Maurice Tremblay, Jean Boucher, Roland Parenteau (ex-directeur général du C.O.E.Q. et de l'O.P.D.Q.)...

Si la rotation des conférenciers est très grande, ce sont surtout des intellectuels reliés au monde de l'éducation, particulièrement des spécialistes des sciences sociales, qui participent aux débats. Outre certains noms mentionnés plus haut, Fernand Dumont, Jean-Charles Falardeau et Arthur Tremblay de l'Université Laval seront parmi ceux-là.

En fait, la plupart des têtes d'affiches de la nouvelle intelligentsia défilent chaque année à l'ICAP. Une bonne partie de l'équipe de Cité Libre, les journalistes vedettes du Devoir et de La Presse de même que certains dirigeants du monde syn- 
dical sont de la partie. De sorte que l'on peut considérer l'ICAP comme l'un des relais importants du réseau des intellectuels.

Le projet de l'Institut est conforme à la pensée démocratique étapiste de toute une génération d'intellectuels contestataires: il s'agit de réunir autour d'un objectif de démocratie élémentaire tout ce que le Québec peut compter alors de «bonnes volontés » et d' « esprits éclairés».

À l'ouverture de la première conférence en 1954, le président Léon Lortie précise bien les intentions du groupe:

«Il existe, quel que soit le régime qui régit un peuple, un ensemble de droits et de devoirs des citoyens afin d'assurer à la fois le bien commun et celui des particuliers (...) En réfléchissant nous-mêmes sur ces questions, nous ne faisons que remplir les obligations que nous imposent nos fonctions d'hommes mêlés à la vie publique. Mais nous avons en même temps conscience de participer à une tâche essentielle qui est de stimuler l'éducation civique et de fonder le patriotisme sur des réalités concrètes et déterminantes ${ }^{22}$.

Non seulement l'Institut veut «révéler l'attitude consciente et inconsciente des Canadiens français» au sujet de l'État et de la démocratie mais il tente également de «situer la question canadienne-française dans son contexte mondial» de manière à élargir nos perspectives de pensée.

Le contenu et la variété des thèmes abordés témoignent de la démarche suivie par les intellectuels de l'ICAP: "Le peuple souverain (La démocratie)» (1954), «L'éducation» (1956), « La communauté mondiale et le Canada» (1957), «L'Église et le Québec» (1960), «Le rôle de l'État» (1962)...

$\mathrm{Au}$ fil des conférences, il s'agit constamment de prendre conscience des défis de la modernisation, de faire l'inventaire de nos carences, l'évaluation de nos besoins et de la somme d'efforts nécessaires pour assurer le développement rationnel de la société québécoise.

22. Léon Lortie, "Présentation de l'Institut», in Institut Canadien des Affaires Publiques. Le peuple souverain, Ste-Marguerite, 1954, 2-3. 
Deux thèmes dominent particulièrement les discussions de l'ICAP: l'éducation et l'État.

On déplore d'abord notre ignorance collective, celle de notre peuple et celle de nos élites, vue comme la première cause du piètre état de notre vie démocratique. Il faut en conséquence refondre de toute urgence notre système d'éducation et le contenu de l'enseignement pour qu'il soit adapté aux exigences de la société moderne. Cela implique que l'on se débarrasse d'un humanisme traditionnel poussiéreux dont la morale fataliste fait de l'individu plus un sujet qu'un citoyen actif maître de son destin. On propose, en lieu et place, un nouveau discours culturel axé sur un savoir concret qui permette réellement de déchiffrer la réalité du progrès technologique et industriel ${ }^{23}$.

La modernisation et la démocratisation de l'enseignement apparaissent pour les intellectuels de l'ICAP comme la clé de notre développement collectif. Celui-ci repose en effet dans leur esprit, sur l'existence d'une véritable élite politique et économique au Québec. Cette idée n'est évidemment pas neuve. Des harangues d'Édouard Montpetit aux discours de nos ministres actuels, elle fait partie du folklore intellectuel national.

Parallèlement au problème de l'éducation, les intellectuels de l'ICAP font l'apologie de l'extension du rôle de l'État dans la société. S'adressant au peuple québécois, ils cherchent à comprendre sa crainte congénitale de l'État. Pourquoi cette peur? se demande-t-on, puisque l'État, lorsqu'il est bien dirigé et qu'il jouit de pouvoirs suffisants, ne peut que contribuer à protéger le «Bien commun» du jeu aveugle des forces économiques capitalistes ${ }^{24}$.

23. Voir particulièrement: Institut Canadien des Affaires Publiques. L'éducation, 1956 et L'utilisation des ressources bumaines, 1965.

24. Voir particulièrement: Institut Canadien des Affaires Publiques. Le rôle de l'État, Montréal Éditions du Jour, 1962. 
On discerne, dans le discours sur l'État, la grande attraction qu'a exercée, à une certaine époque, l'expérience du Plan en France. La planification étatique est considérée comme essentielle dans le contexte québécois, particulièrement dans la rationalisation du développement des ressources humaines.

Les deux problèmes centraux que sont l'éducation et l'extension du rôle de l'État se trouvent intimement liés dans la logique des conférenciers de l'ICAP. En effet, comme l'indique André Raynauld en 1962, l'intervention de l'État ne sera positive que si les gouvernants possèdent une expertise technique et scientifique suffisante pour l'orienter de manière rationnelle ${ }^{25}$.

Par ailleurs, aucune tentative de planification ne pourra réussir sans la participation active de la population. Au milieu des années soixante, Guy Coulombe, aménagiste-en-chef du Bureau d'Aménagement de l'Est du Québec (BAEQ), l'un des premiers organes de planification au Québec, va exposer le problème:

«Après les premiers inventaires de base, il est apparu évident au BAEQ que le problème de l'éducation était crucial par rapport au développement régional. En effet, le développement de certains secteurs de l'activité économique allait exiger une mobilité occupationnelle que rendait impossible le bas niveau d'éducation... ${ }^{26}$.

C'est, en quelque sorte, un modèle de société technocratique que les intellectuels de l'ICAP défendent auprès de la population québécoise par le biais d'une certaine «éducation civique et démocratique». On voit, en effet, se dessiner à travers le discours global tenu pendant treize ans, les bases d'une société moderne où doivent dominer les impératifs de "Rendement», «Efficacité», et «Rationalité » par opposition au dé-

25. André Raynauld, "Présentation", in Ibid., 9-11.

26. Guy Coulombe, «Pour une véritable politique de développement socio-économique ", Institut Canadien des Affaires Publiques. L'utilisation des ressources bumaines, op. cit., 91-92. 
veloppement anarchique que la société traditionnelle québécoise a toujours connu.

Après l'avoir diffusé par divers moyens auprès de la population, les intellectuels d'après-guerre tenteront de concrétiser leur projet de société. Ce sera alors le début de toute l'aventure de la planification au Québec durant les années soixante.

«Créer des rôles..."

Les intellectuels d'après-guerre ne sont pas qu'organiques. Leur action ne se résume pas qu'à la seule médiatisation des intérêts du groupe social dominant. De par leurs fonctions et leur formation communes, ils forment une catégorie sociale relativement homogène. Ainsi, ont-ils la même aspiration à conserver le pouvoir que leur confere le savoir.

Or, en mettant en relief l'importance du développement de la connaissance, ils tendent, consciemment ou pas, à légitimer leur propre position de force dans la société. En se faisant prophètes de la modernisation durant l'après-guerre et la Révolution tranquille, les «nouveaux intellectuels» travaillaient à réunir les conditions favorables à leur propre ascension sociale.

Singulièrement, c'est dans le cadre d'une conférence de l'ICAP sur le rôle de l'État en 1962 que l'on fait la meilleure description des aspirations des spécialistes des sciences sociales appliquées. Au tout début de la Révolution tranquille, Jean Boucher (ci-devant fonctionnaire au ministère fédéral de la citoyenneté et de l'immigration) déclare:

"Aujourd'hui, les Écoles de sciences sociales se trouvent associées à de vastes programmes de transformation sociale qui leur ouvrent soudain, au-delà de la simple observation, le Terre promise de l'expérimentation scientifique. La prospection des façons disciplinées de poursuivre le progrès social devient aussi une étape essentielle de leur démarche scientifique. Elles se prennent alors à explorer, sous le couvert de l'organisation communautaire, de la planification régionale, de l'assistance technique, divers ensembles interdisciplinaires qui de- 
vraient les mener, dans un avenir prochain, à un type élémentaire d'ingénieur social, d'agent de la transformation sociale. »

Bouclant le pronostic, Boucher ajoute que ce sont les gouvernements qui, les premiers, auront à éprouver et à donner la consécration professionnelle à cet intellectuel. Jusqu'ici, on ne savait, en effet, que «laisser faire». On découvre aujourd'hui, selon Boucher, la façon «d'aider à faire» ou «d'aider à s'aider». «L'art politique nouveau ne consiste plus uniquement à régler ou à occuper des rôles: il consiste encore et surtout à créer des rôles, à en favoriser et à en épauler l'exercice ${ }^{27}$.

\section{Conclusion}

C'est en jouant un rôle d'éducateur populaire que l'intellectuel s'engage dans la voie du changement politique durant l'après-guerre. Fort du savoir spécialisé qu'il a acquis au contact des nouvelles disciplines des sciences sociales, il contribue à la prise de conscience collective qui devait mener la société québécoise aux grandes réformes de la Révolution tranquille.

Dans le cadre de son entreprise d'éducation civique, l'intellectuel œuvre, de manière spécifique, à l'intérieur d'un réseau assez bien défini d'institutions parmi lesquelles certains médias, la télévision en particulier, s'imposent par leur pouvoir de diffusion auprès du grand public. Aussi, la Société RadioCanada, avec des émissions d'affaires publiques comme celles de l'ICAP, constitue, durant les années cinquante, un relai important dans la propagation du message démocratique.

Mais de façon peut-être plus concrète, ce « réseau de résistance » a constitué un tremplin pour l'ascension sociale de toute une nouvelle catégorie d'élites. Les organisations qui la composaient ont fourni une première tribune aux idées de la généra-

27. Jean Boucher, «La fonction publique», Institut Canadien des Affaires Publiques. Le rôle de l'État, op. cit., 98-99. 
tion montante. Elles lui ont ainsi permis de se forger une légitimité et un pouvoir intellectuels auprès de larges secteurs de la société.

L'existence d'un réseau aussi bien défini d'organisations d'intellectuels ne pouvait, bien sûr, s'inscrire que dans une conjoncture historique et politique exceptionnelle. La précarité de la situation de l'opposition sous Duplessis commandait aux progressistes, peu nombreux, de resserrer leurs rangs.

C'est la raison pour laquelle des conférences comme celles de l'ICAP ont pu avoir lieu. Il ne sera jamais plus possible, à partir du milieu des années soixante, de réunir autant d'intellectuels provenant de secteurs d'intérêt et d'opinion aussi divers. Comment peut-on croire, par exemple, qu'il soit possible encore aujourd'hui de réunir des hommes aussi intellectuellement opposés que Marcel Rioux et Marc Lalonde?

Le fait est qu'avec la Révolution tranquille, la «démocratie minimale» tant souhaitée par une certaine génération d'intellectuels était conquise. Dès lors, la société québécoise allait connaître un éclatement de ses structures sur le plan idéologique. Le duplessisme étant abattu, les diverses tendances de la démocratie pouvaient s'affirmer pleinement. Parallèlement au débat sur la question nationale, les diverses factions de la catégorie montante (technocrates du secteur privé et technocrates du secteur public) après avoir «pris leur place» dans la société, devaient s'opposer sur la question de l'importance à accorder au rôle de l'État au Québec.

Ainsi, il devenait littéralement impossible de recréer le climat de collaboration inter-groupes de l'après-guerre. La société québécoise répondait désormais au mot d'ordre de Gérard Pelletier: «Feu l'unanimité!». 